\title{
Editorial: special issue on ubiquitous electronic commerce systems
}

\author{
Robert H. Deng · Jari Veijalainen • Shiguo Lian • \\ Dimitris Kanellopoulos
}

Published online: 29 October 2010

(C) Springer Science+Business Media, LLC 2010

\section{Introduction}

Ubiquitous computing is a post-desktop model of human-computer interaction in which information processing has been thoroughly integrated into everyday objects and activities. Emerging ubiquitous electronic commerce systems (UECS) are expected to be available anytime, anywhere, and using different official or personal computing devices. Systems and services such as digital libraries, on-line business transactions, mobile office and mobile TV are widely deployed. Users will be able to access these services anytime, anywhere, while using any computing device in a pervasive way. For example, a user may bring a PDA on a field trip, carry a laptop (with both wireless and wired network cards) on a business trip, use high performance workstations at work, and use desktop PCs at home (with dial-up, cable, or DSL connection). In another example, a user continues to watch the soccer game over home $\mathrm{TV}$, while he watches the game through a mobile device out of home. Some other

\footnotetext{
R.H. Deng $(\varangle)$

Singapore Management University, Singapore, Singapore

e-mail: robertdeng@smu.edu.sg

J. Veijalainen

University of Jyväskylä, Jyväskylä, Finland

e-mail: veijalai@cs.jyu.fi

S. Lian

France Telecom (Orange Labs) Beijing, Beijing, China

e-mail: shiguo.lian@ieee.org

D. Kanellopoulos

University of Patras, Patras, Greece

e-mail: d_kan2006@yahoo.gr
} 
ubiquitous services include mobile games and entertainment, mobile blog, mobile community, home network, and so on.

Since ubiquitous electronic commerce systems (UECS) or services are constructed on the convergence of various networks (wired or wireless networks, broadcasting or multicasting networks, broadband or band-limited networks, etc.) and devices (PC, PDA, Cell Phone, TV, Camera, etc.), the new challenge in ubiquitous commerce management is to deal with heterogeneous client capabilities and dynamic end-to-end network and system resources availability, and to ensure satisfactory quality of service (QoS) support to each client. Additionally, privacy, security and ethical issues are becoming increasingly important topics in ubiquitous computing.

This special issue aims to expose the readership to the latest research results on ubiquitous electronic commerce systems (UECS). It is composed of 5 refereed papers covering such topics as location-based services, secure ubiquitous multimedia sharing, intelligent multimedia services, and privacy preserving in commercial networks. The issue is expected to demonstrate pioneer work in this field, investigate the novel solutions and discuss the future trends in this field.

\section{The papers in this special issue}

The first paper, "Location-Based Services Deployment and Demand: A Roadmap Model" by K. Petrova and B. Wang, investigates the popular topic of Location-based Services (LBS). LBS benefits from the advancements in mobile, wireless and positioning technologies, e.g., route guiding and emergency call-out assistance. This paper not only reviews the latest development and deployment of LBS in different geographic areas (e.g., USA, EU, Japan, South Korea and New Zealand), but also analyzes such issues as regulatory environment and customer demand, and furthermore, proposes the roadmap of LBS development. Thus, readers can not only obtain the information about latest LBS, but also get suggestions on the future research and work in LBS.

The second paper, "Towards the Regulation of Ubiquitous Mobile Government: A Case Study on Location-Based Emergency Services in Australia" by A. Aloudat and K. Michael, investigates the issues in mobile government that establishes mobile alerts and location-based emergency warning systems. Based on Australian national emergency warning system (NEWS), the qualitative results are given to show some major issues faced by government, business and society in order to realize a fully fledged system for personal mobile devices. Additionally, the complex interplay between government agencies, telecommunications carriers and the Australian public is presented. The paper is expected to provide some valuable advices to design mobile government applications.

In the third paper, "Innovative Internet Video Consuming Based on Media Analysis Techniques" by, S. Lian, innovative Internet video services are investigated, e.g., TV program preview, personalized browsing, content based recommendation, and ubiquitous TV services. Additionally, such multimedia analysis techniques as text analysis, image analysis and video analysis are also introduced, which make innovative services available. Furthermore, some innovative user experiences based on 
media analysis are presented. Finally, the paper discusses some open issues and potential research topics in media analysis and the related applications in Internet video services. Readers are expected to get valuable information about intelligent and ubiquitous TV services.

The fourth paper, "Service and P2P based Secure Media Sharing in Mobile Commerce Environments" by X. Chen and S. Lian, investigates the P2P (Peer-to-Peer) based media sharing in mobile commerce environments. It proposes a secure mobile media service system, which is able to trace illegal distributors in $\mathrm{m}$-commerce applications. In this scheme, the decryption operation and fingerprint embedding operation are combined together, which avoids the leakage of plain media content in mobile transfer. Additionally, these operations are implemented by the peer, which makes the scheme compliant with existing Peer-to-Peer Digital Rights Management (DRM) systems and very suitable for secure media distribution in m-commerce. Both the architectures and modes are presented and discussed. It will provide valuable information to researchers or engineers working in security aspect of mobile commerce.

In the fifth paper, "A Privacy Policy Conflict Detection Method for Multi-owner Privacy Data Protection" by Y. Ren et al., investigates the privacy issues in social networks, including ubiquitous commerce system. Current privacy-preserving works focus on single-owner privacy data, while multi-owner privacy data that are often popular in practice are not considered. This paper introduces the characteristics of multi-owner privacy data and its protection requirement. Then, a data schema based on deputy mechanism for multi-owner privacy data is proposed. Based on the schema, a privacy policy conflict detection method is proposed. This method models the privacy policy and each possible policy conflict pattern based on sub-graph isomorphic, and provides the corresponding algorithm to detect them. Since privacy becomes more and more important in ubiquitous electronic commerce system, this paper is expected to give some interesting research topics and valuable suggestions to researchers or engineers.

Acknowledgements The guest editors wish to thank Prof. Bezalel Gavish for providing the opportunity to edit this special issue on Ubiquitous Electronic Commerce Systems, and Ms. Jackie James and other editors for providing latest publishing information and making this issue published. We would also like to thank the authors for submitting their works as well as the referees who have critically evaluated the papers. Finally, we hope the reader will share our joy and find this special issue very useful.

Robert H. Deng received his B.Eng. from National University of Defense Technology, Changsha, China, M.Sc. and Ph.D. from Illinois Institute of Technology, Chicago, USA. He is Professor of Information Systems at Singapore Management University (SMU). He is also Adjunct Professor at Wuhan University, Zhejiang University, and Jinan University. His current research interests include applied cryptography, data and multimedia security, wireless and sensor network security, and trusted computing. He is the inventor of 26 patents, some of them have been incorporated into commercial products. He has more than 250 papers in refereed conferences and journals. He served as general chair, program committee chair and PC member of numerous international conferences. He is also Associate Editor of the IEEE Transactions on Information Forensics and Security, Associate Editor of Security and Communication Networks Journal (John Wiley), and Area Editor of Journal of Computer Science and Technology (Science Press, China and Springer). Prior to 2004, most of his working career was with research institutes. He was Research Staff Member and Project Leader of Communications Group at the Institute of Systems Science (07/198706/1991), Senior Lecturer with the Department of Electrical Engineering at National University of Singapore (07/1991-06/1994), Senior Research Staff Member and Manager of Information Security Group at Institute of Systems Science (07/1994-03/1998), Principal Research Staff Member, Deputy Director and 
then Director of Ubiquity Lab at Kent Ridge Digital Labs (04/1998-12/2001), Principal Research Staff Member and Director of Ubiquitous and Distributed Computing Program at Labs for Information Technology, and Principal Scientist and Manager of Infocomm Security Department at Institute for Infocomm Research (11/2002-07/2004).

Jari Veijalainen is working as a full professor at the department since August 1996. On Nov. 17, 1999 he was nominated to a permanent full professor position by the University of Jyvaskyla. Dr. Veijalainen has taken part in various EU-financed projects. He is currently a Member of the Editorial Board of the VLDB Journal, and a member of the Editorial Board of Wireless Networks (WINE). He has served on tens of international program committees, among them VLDB'96, VLDB'01, ICDE'01, ICDE'06, ICDE'07, ICCCN'02-05,'07 and refereed papers for major journals and conferences including IEEE Data Engineering, Very Large Databases, IEEE Data\&Knowledge Engineering, VLDB Journal, and ACM Transactions on Databases, ACM Monet. Since 1995 he has evaluated projects and project proposals for the European Commission. He has published one book and is a co-editor in another book. In all, he has published tens of scientific conference and journal papers and several chapters in scientific monographs. Dr. Veijalainen is a voting member of ACM, and a member of IEEE Computer Society and the Finnish Information Processing Association. His research interests include heterogeneous transaction management, transaction models and mechanisms for computer supported co-operative work, workflow systems and electronic commerce systems, formal modeling, mobile computing, software engineering, and mobile multimedia data management.

Shiguo Lian got his Ph.D. from Nanjing University of Science and Technology, China. He was a research assistant in City University of Hong Kong in 2004. Since July 2005, he has been a Research Scientist with France Telecom R\&D (Orange Labs) Beijing. He is the author or co-author of more than 80 refereed international journal and conference papers covering topics of secure multimedia communication, intelligent multimedia services, and ubiquitous communication. He has contributed 15 book chapters and held 16 filed patents. He authored the book "Multimedia Content Encryption: Techniques and Applications" (CRC Press, 2008), and edited 5 books. He got the Nomination Prize of "Innovation Prize in France Telecom" and "Top 100 Doctorate Dissertation in Jiangsu Province" in 2006. He is a member of IEEE Communications \& Information Security Technical Committee, IEEE Multimedia Communications Technical Committee, and IEEE Technical Committee on Nonlinear Circuits and Systems. He is on the editor board of several international journals. He is the guest editor of more than 10 international journals. He is in the organization committee or the TPC member of refereed conferences, including IEEE ICC2008/2009/2010, IEEE GLOBECOM2008/2009/2010, IEEE CCNC2009, IEEE ICCCN2009, etc. He is also the reviewer of refereed international magazines and journals.

Dimitris Kanellopoulos is a member of the Educational Software Development Laboratory (ESDLab) in the Dept of Mathematics at the University of Patras, Greece. He is also a lecturer in the Dept of Tourism Management at the Technological Educational Institute of Patras. He received a diploma in electrical engineering and a Ph.D. in multimedia communications from the University of Patras. Since 1990, he was a research assistant in the Dept of Electrical and Computer Engineering at the University of Patras and involved in several EU R\&D projects. His research interests include multimedia communications, Webbased education, intelligent information systems, semantic Web, and knowledge representation. He has many publications to his credit in International Journals and Conferences at these areas. He serves as a member of the editorial boards in ten academic journals. 